\title{
On the Number of Leaders Needed to Ensure Network Connectivity in Arbitrary Dimensions
}

\author{
Tove Gustavi, Dimos V. Dimarogonas, Magnus Egerstedt and Xiaoming $\mathrm{Hu}$
}

\begin{abstract}
We examine the leader-to-follower ratio needed to maintain connectivity in a leader-follower multi-agent network with proximity based communication topology. The paper extends the one-dimensional results of [2] to the two dimensional case. In the scenario we consider, only the leaders are aware of the global mission, which is to converge to a known destination point. Thus, the objective of the leaders is to drag the team to the desired goal. In the paper we obtain bounds on the number of leaders needed to complete the task. The results are first established for an initially complete communication graph and then extended to the incomplete case. Computer simulations support the derived theory.
\end{abstract}

\section{INTRODUCTION}

Distributed control of multi-agent systems is an important issue in a number of applications, including multi-agent robotics [5], networked sensor and health maintenance [6] and formation control [4],[1] just to name a few. One way in which the user can interact with such systems is through so-called leader agents, whose dynamics need not conform to those of the follower agents. In this paper we study such systems, i.e., systems where a selected subset of the agents are following a task-level controller encoding the transport of the network from one location to another. The rest of the agents have no notion of these task-level objectives, and are instead executing a local interaction-based control strategy for keeping the team together. The reason why such a heterogeneous network configuration is desirable is that it frees up resources by only insisting on a select subset of agents being able to tell global positions and/or positions relative to particular landmarks, thus limiting the required sensor load of the remaining agents. This was for instance the case in [9], in which a collection of mobile sensor nodes were to traverse long distances before assembling the desired sensing configuration.

The reasons for prescribing networked solutions to engineering systems range from cost considerations (many cheap systems for solving a problem rather than a single expensive system) to strength-in-numbers arguments. However, as of yet, few studies have addressed the question concerning how many agents one actually needs. In this paper, we pursue

T. Gustavi and X. Hu are with the School of Engineering Sciences, Royal institute of Technology (KTH), 10044 Stockholm, Sweden \{gustavi, hu\}@math.kth.se

D. V. Dimarogonas is with the Laboratory for Information and Decision Systems, Massachusetts Institute of Technology, Cambridge, MA, U.S.A. \{ddimar@mit.edu\}

M. Egerstedt is with the School of Electrical and Computer Engineering, Georgia Institute of Technology, Atlanta, GA 30332, USA magnus@ece.gatech.edu

An extended journal version of this work has been submitted to Automatica. this question in the context of a leader-follower network. In particular, we ask the question "How many leaders do you really need?". While many issues regarding controllability and stability of leader-follower networks have been addressed recently in, for instance, [10],[8], and connectivity maintenance for homogeneous multi-agent systems was examined for example in [4], the issue of how the ratio of leaders-tofollowers affects connectivity is a novel topic, introduced by the authors in [2].

In this paper we take a geometric approach to the problem. We show that by considering the derivative of the distance between two agents it is possible to obtain rules for how to select the relative number of leaders-to-followers and the goal attraction function of the leader agents so that connectivity and convergence to a common goal position are guaranteed. Preliminary results for agents moving in one dimension were obtained in the conference paper [2]. The current paper extends the results to the case of $n$ dimensions. In particular we study the case $n=2$.

The rest of the paper is organized as follows: in Section II we introduce the considered framework and present the approach. In Sections III and IV we demonstrate the proposed approach on two examples of connected networks and in Section $\mathrm{V}$ the theoretical results are illustrated in simulations. Finally, in Section VI, the results of the paper are summarized.

\section{System and Problem Statement}

Consider $N$ agents evolving in $\mathbb{R}^{n}$. We use single integrator agents whose motions obey the model: $\dot{x}_{i}=u_{i}, i \in \mathcal{N}=$ $\{1, \ldots, N\}$. The agents belong either to the subset of leaders $\mathcal{N}^{l}$, or to the subset of followers, $\mathcal{N}^{f}$, such that $\mathcal{N}^{l} \cup \mathcal{N}^{f}=$ $\mathcal{N}$ and $\mathcal{N}^{l} \cap \mathcal{N}^{f}=\emptyset$. The number of agents in each set is given by $\left|\mathcal{N}^{f}\right|=N_{f}$ and $\left|\mathcal{N}^{l}\right|=N_{l}$ respectively. Due to shortcomings of the sensors, each agent has a limited sensing zone of radius $\Delta>0$. At any given time, the agents located within the sensing zone of agent $i \in \mathcal{N}$ are referred to as the neighbors of agent $i$. Define $\mathcal{N}_{i}=\left\{j \in \mathcal{N}:\left|x_{i}-x_{j}\right| \leq \Delta\right\}$. The set $\mathcal{N}_{i}$ is called agent $i$ 's neighboring set and is updated every time an agent enters/leaves the sensing zone of agent $i$. We denote $\left|\mathcal{N}_{i}\right|=N_{i}$.

Each agent has knowledge of the relative coordinates of all its neighbors, but can not detect or communicate with agents outside its sensing zone. To keep track of the active communication links we introduce a communication graph, $\mathcal{G}=\{V, E\}$, that describes the group topology. $\mathcal{G}=\{V, E\}$ consists of a set of vertices $V=\{1, \ldots, N\}$ indexed by the team members, and a set of edges, $E=\{(i, j) \in$ 
$\left.V \times V \mid i \in \mathcal{N}_{j}\right\}$ containing pairs of vertices that represent inter-agent communication links. Since the set $E$ is time varying, the graph $\mathcal{G}=\mathcal{G}(t)$ itself, is time-varying. In the following sections we will establish sufficient conditions for some particular networks of agents to stay connected. We start by introducing a notation for the distance between two arbitrary agents $i$ and $j$. Let $\delta_{i j}=\delta_{j i}=\left|x_{i}-x_{j}\right|=$ $\sqrt{\left(x_{i}-x_{j}\right)^{T}\left(x_{i}-x_{j}\right)} \geq 0$. Then

$$
\frac{d \dot{\delta}_{i j}^{2}}{d t}=2 \delta_{i j} \dot{\delta}_{i j}=2\left(x_{i}-x_{j}\right)^{T}\left(\dot{x}_{i}-\dot{x}_{j}\right) .
$$

Unlike $\dot{\delta}_{i j}, \frac{d \delta_{i j}^{2}}{d t}$ is defined on all of $\mathbb{R}^{n}$. The function has always the same sign as $\dot{\delta}_{i j}$. Hence, a sufficient condition for agent $i$ and $j$ to remain connected is $\frac{d \delta_{i j}^{2}}{d t} \leq 0$ when $\delta_{i j}=\Delta$.

The dynamics of the followers are given by the standard consensus equation [7], meaning that each follower moves in the direction of the average position of its neighbors. For $i \in \mathcal{N}^{f}$ we have:

$$
\dot{x}_{i}=-\sum_{k \in \mathcal{N}_{i}}\left(x_{i}-x_{k}\right)=-N_{i} x_{i}+\sum_{k \in \mathcal{N}_{i}} x_{k} .
$$

The dynamics of the leaders include an additional attraction term which aims at dragging the team to a pre-defined goal position $x=d$. For agent $i$, define $\delta_{i}=\left|d-x_{i}\right|$. The dynamics for an arbitrary leader $i \in \mathcal{N}^{l}$ are given by

$$
\begin{aligned}
\dot{x}_{i} & =-\sum_{k \in \mathcal{N}_{i}}\left(x_{i}-x_{k}\right)+F\left(x_{i}, d\right) \\
& =-N_{i} x_{i}+\sum_{k \in \mathcal{N}_{i}} x_{k}+F\left(x_{i}, d\right),
\end{aligned}
$$

where $F\left(x_{i}, d\right)$ is the goal attraction function

$$
F\left(x_{i}, d\right)= \begin{cases}f\left(\delta_{i}\right) \frac{d-x_{i}}{\delta_{i}} & \delta_{i}>0 \\ 0 & \delta_{i}=0\end{cases}
$$

At any $x_{i} \neq d$, the direction of $F\left(x_{i}, d\right)$ is towards the goal and the magnitude is decided by the continuous scalar function $f\left(\delta_{i}\right) \geq 0 . f\left(\delta_{i}\right)$ is depending only on agent $i$ 's distance to $d$ and can be designed to suit the application. The continuity of $F\left(x_{i}, d\right)$ is guaranteed by requiring $f(0)=$ 0 and $\lim _{\delta^{+} \rightarrow 0} \frac{f(\delta)}{\delta}<\infty$, so that $\lim _{x^{+} \rightarrow d} F(x, d)=$ $F(d, d)=0$. A simple example of a possible goal attraction function that satisfies these requirements is $f(\delta)=\delta$.

The following Lemma guarantees the boundedness of solutions of the closed-loop system:

Lemma 1: Let $\mathcal{G}$ be a nonempty graph consisting of followers and leaders with dynamics decided by (2) and (3). Define $\Omega$ to be the convex hull of the agents in $\mathcal{G}$ and the goal position $d, \Omega=\operatorname{Co}(\mathcal{G} \cup d)$, and let $\Omega_{0}$ denote the convex hull at time $t=0$. Now assume that none of the agents in $\mathcal{G}$ are connected to any agents $x_{k}, k \notin \mathcal{G}$, and that $f(|d-x|) \leq f_{\max }<\infty$ for all $x \in \Omega_{0}$. Then the trajectories of all agents in $\mathcal{G}$ will remain within $\Omega_{0}$ as $t \rightarrow \infty$.

Proof: We will show that for an arbitrary agent $i \in \mathcal{G}$, positioned on the boundary of $\Omega$, the motion is either on the boundary of $\Omega$ or pointing inside the polytope $\Omega$.
If $i \in \mathcal{N}^{f}$ the motion is given by $\dot{x}_{i}=-\sum_{k \in \mathcal{N}_{i}}\left(x_{i}-x_{k}\right)$. If $N_{i}=0$ the agent will not move at all and the proof is trivial. Now consider the case $N_{i}>0$. By setting $\alpha=$ $N_{i}^{-1}$ and rearranging the terms we can show that $\alpha \dot{x}_{i}=$ $-x_{i}+\sum_{k \in \mathcal{N}_{i}} \frac{x_{k}}{N_{i}}$. Apparently the motion of follower $i$ is directed towards the barycenter of the subgraph $\mathcal{N}_{i} \subseteq \mathcal{G}$, which, thanks to convexity, is known to lie either on the boundary or in the interior of $\Omega$. By convexity we conclude that the motion of follower $i$ must lie within $\Omega$.

For $i \in \mathcal{N}^{l}$, we have $\dot{x}_{i}=-\sum_{k \in \mathcal{N}_{i}}\left(x_{i}-x_{k}\right)+f(\mid d-$ $\left.x_{i} \mid\right) \frac{d-x_{i}}{\left|d-x_{i}\right|}$. If $N_{i}=0$ the agent will, depending on the magnitude of $f\left(\left|d-x_{i}\right|\right)$, either not move at all, or move directly towards $d$. Since $d$ is in $\Omega$ and since $\Omega$ is convex, we see that in either case agent $i$ will remain in $\Omega$. If $N_{i}>0$ we define $\beta=\left(N_{i}+f\left(\left|d-x_{i}\right|\right)\right)^{-1}$. Then we get: $\beta \dot{x}_{i}=-x_{i}+\beta\left(N_{i} \sum_{k \in \mathcal{N}_{i}} \frac{x_{k}}{N_{i}}+f\left(\left|d-x_{i}\right|\right) d\right)$. The motion of $i$ is directed towards a convex combination of the barycenter of $\mathcal{N}_{i} \subseteq \mathcal{G}$ and the goal $d$. By definition, this convex combination lies within the convex hull of $\mathcal{G} \cup d$, and therefore, by the convexity of $\Omega$, agent $i$ stays in $\Omega$.

Since the motion of any agent on the boundary of $\Omega$ is either on the boundary of $\Omega$ or directed into the interior of $\Omega$, we can conclude that no agent will ever enter outside the convex hull defined by the initial positions of the agents and the goal $d$. Hence, $\Omega_{0}$ is an invariant set. $\diamond$

The next Theorem states that the closed-loop system converges to the goal position $d$ if the communication graph remains connected:

Theorem 2: Let the closed loop dynamics (2) and (3). Let $x(0) \in \Omega$ and assume that the communication graph $\mathcal{G}(t)$ remains connected. Then, $\lim _{t \rightarrow \infty} x_{i}(t)=d$ for all $i \in \mathcal{N}$. Proof: Equations (2) and (3) are written in stack vector form as $\dot{x}=-\left(L \otimes I_{2}\right) x-F(x, d)$, where $x=\left[x_{1}, \ldots, x_{N}\right]^{T}$, and the elements of the vector $F(x, d)$ are given by $F_{i}(x, d)=0$, if $i \in \mathcal{N}^{f}$ and $F_{i}(x, d)=F\left(x_{i}, d\right)$, if $i \in \mathcal{N}^{l}$, and where $L$ is the Laplacian of $\mathcal{G}(t)$. The eigen-properties of the Laplacian matrix are well established in the cooperative control literature and are not recapped here. The reader is referred to [3] for a review of the Laplacian matrix properties.

Defining $z=x-d$, with $d=[d, \ldots, d]^{T}$ we have $\dot{z}=$ $-\left(L \otimes I_{2}\right) x-F(x, d)=-\left(L \otimes I_{2}\right)(z+d)-F(x, d)=$ $-\left(L \otimes I_{2}\right) z-F(z)$, where now the vector $F(z)$ is given by $F_{i}(z)=0$, if $i \in \mathcal{N}^{f}$, and $F_{i}(z)=\delta_{i} f\left(\delta_{i}\right)=\left|z_{i}\right| f\left(\left|z_{i}\right|\right)$. Taking $V=\frac{1}{2} z^{T} z$ as a candidate Lyapunov function we get $\dot{V}=\dot{z} z^{T}=-z^{T}\left(L \otimes I_{2}\right) z-z^{T} F(z)=-z^{T}\left(L \otimes I_{2}\right) z-$ $\sum_{i \in \mathcal{N}^{l}} \delta_{i} f\left(\delta_{i}\right)$, which is negative semidefinite due to the eigen-properties of $L$ and $f$ being monotonically increasing. By LaSalle's Invariance Principle, we have that $\delta_{i} \rightarrow 0$ for all $i \in \mathcal{N}^{l}$. Now, due to the first term we also have that $z^{T}\left(L \otimes I_{2}\right) z \rightarrow 0$ so that all elements of $z$ attain an equal value. This means that $\left|z_{i}\right| \rightarrow z^{*}$ or $\delta_{i} \rightarrow z^{*}$ for all $i \in \mathcal{N}$. Since $\delta_{i} \rightarrow 0$ for all $i \in \mathcal{N}^{l}$ we finally get $z^{*}=0$, i.e., $\delta_{i} \rightarrow 0$ for all $i \in \mathcal{N} . \diamond$

By virtue of the above Theorem, agents converge to the desired goal configuration as long as the communication graph remains connected throughout the evolution of the 
closed-loop system. In the following sections, we establish conditions under which connectivity is preserved for some classes of initially connected graphs.

\section{COMPlete Graph CASE}

In this section, we assume that all agents are initially within the sensing zone of one another, i.e., at a distance less than $\Delta$ from each other. Hence, the initial graph $\mathcal{G}(t)$ is complete and of course, connected. In the sequel, we derive sufficient conditions for the graph to remain complete.

Since the graph is initially complete, the dynamics for the follower agents are given by:

$$
\begin{aligned}
\dot{x}_{i} & =-\sum_{k \in \mathcal{N}^{f}}\left(x_{i}-x_{k}\right)-\sum_{k \in \mathcal{N}^{l}}\left(x_{i}-x_{k}\right) \\
& =-N_{f} x_{i}+\sum_{k \in \mathcal{N}^{f}} x_{k}-N_{l} x_{i}+\sum_{k \in \mathcal{N}^{l}} x_{k} \\
& =-N x_{i}+\sum_{k \in \mathcal{N}} x_{k}, \quad \forall i \in \mathcal{N}^{f} .
\end{aligned}
$$

Similarly, the dynamics for the leader agents are given by:

$$
\dot{x}_{i}=-N x_{i}+\sum_{k \in \mathcal{N}} x_{k}+F\left(x_{i}, d\right), \quad \forall i \in \mathcal{N}^{l} .
$$

Follower-follower connections: For two arbitrary followers $i, j \in \mathcal{N}^{f},(1)$ and (5) give $\frac{d \delta_{i j}^{2}}{d t}=2\left(x_{i}-x_{j}\right)^{T}\left(\dot{x}_{i}-\dot{x}_{j}\right)=$ $2\left(x_{i}-x_{j}\right)^{T}\left[-N x_{i}+\sum_{k \in \mathcal{N}} x_{k}+N x_{j}-\sum_{k \in \mathcal{N}} x_{k}\right]=$ $-2 N \delta_{i j}^{2}$. We see that $\delta_{i j}^{2} \rightarrow 0$. In particular, this implies that $\frac{d \delta_{i j}^{2}}{d t}<0$ when $\delta_{i j}$ tends to $\Delta$, so we can conclude that if the two followers are initially within each others sensing zones, they will remain connected as $t \rightarrow \infty$.

Leader-leader connections: For two arbitrary leaders $i, j \in \mathcal{N}^{f}$, (1) and (6) give $\frac{d \delta_{i j}^{2}}{d t}=-2 N \delta_{i j}^{2}+2\left(x_{i}-\right.$ $\left.x_{j}\right)^{T}\left[F\left(x_{i}, d\right)-F\left(x_{j}, d\right)\right]$. Noting that $\left(x_{i}-x_{j}\right)=-((d-$ $\left.\left.x_{i}\right)-\left(d-x_{j}\right)\right)$ makes it possible to re-write the equation for the general case where $x_{i} \neq d, x_{j} \neq d$ : $\frac{d \delta_{i j}^{2}}{d t}=$ $-2 N \delta_{i j}^{2}+2\left(x_{i}-x_{j}\right)^{T}\left[f\left(\delta_{i}\right) \frac{d-x_{i}}{\delta_{i}}-f\left(\delta_{j}\right) \frac{d-x_{j}}{\delta_{j}}\right]=-2 N \delta_{i j}^{2}-$ $2\left[f\left(\delta_{i}\right) \delta_{i}+f\left(\delta_{j}\right) \delta_{j}-\left(\frac{f\left(\delta_{i}\right)}{\delta_{i}}+\frac{f\left(\delta_{j}\right)}{\delta_{j}}\right)\left(d-x_{i}\right)^{T}\left(d-x_{j}\right)\right]$. In the worst-case we have $\left(d-x_{i}\right)^{T}\left(d-x_{j}\right)=\delta_{i} \delta_{j}$, which inserted in the equation for $\frac{d \delta_{i j}^{2}}{d t}$ gives $\frac{d \delta_{i j}^{2}}{d t}=-2 N \delta_{i j}^{2}-$ $2\left[f\left(\delta_{i}\right) \delta_{i}+f\left(\delta_{j}\right) \delta_{j}-f\left(\delta_{i}\right) \delta_{j}-f\left(\delta_{j}\right) \delta_{i}\right]$, so that $\frac{d \delta_{i j}^{2}}{d t}=$ $-2 N \delta_{i j}^{2}-2\left(f\left(\delta_{i}\right)-f\left(\delta_{j}\right)\right)\left(\delta_{i}-\delta_{j}\right)$. It is easy to see from the previous equation that $\frac{d \delta_{i j}^{2}}{d t} \leq 0$ for all inter-agent distances, and for all of $N_{f}$ and $N_{l}$, if we require $f(\delta)$ to be a monotonically increasing function,

$$
f^{\prime}(\delta) \geq 0, \quad \forall \delta \geq 0 .
$$

In other words, any two leaders that are initially connected will remain so if condition (7) is satisfied.

Leader-follower connections: For the case $i \in \mathcal{N}^{f}, j \in$ $\mathcal{N}^{l}$, we have $\frac{d \delta_{i j}^{2}}{d t}=-2 N \delta_{i j}^{2}-2\left(x_{i}-x_{j}\right)^{T} F\left(x_{j}, d\right)$. From the equation above it is easy to see that if $x_{j}=d$. i.e., if the leader is located in $d$ and the corresponding goal attraction term is zero, then $\frac{d \delta_{i j}^{2}}{d t} \leq 0$. If $x_{j} \neq d$ we get $\frac{d \delta_{i j}^{2}}{d t}=$ $-2 N \delta_{i j}^{2}-2 f\left(\delta_{j}\right)\left(x_{i}-x_{j}\right)^{T} \frac{\left(d-x_{j}\right)}{\delta_{j}} \leq-2 N \delta_{i j}^{2}+2 f\left(\delta_{j}\right) \delta_{i j}$, since $-\delta_{i j} \delta_{j} \leq\left(x_{i}-x_{j}\right)^{T}\left(d-x_{j}\right) \leq \delta_{i j} \delta_{j}$. We will now make use of Lemma 1. Let $f_{\max }$ be the largest value that $f\left(\delta_{j}\right)$ assumes within the convex hull defined by the agents initial positions and the goal position $d$. Then $\left|f\left(\delta_{j}\right)\right| \leq f_{\max }$ for all $t \geq 0$ and

$$
f_{\max } \leq N \Delta
$$

is a sufficient condition to guarantee that $\frac{d \delta_{i j}^{2}}{d t} \leq 0$ if $\delta_{i j}=\Delta$, i.e. that any initially connected pair consisting of one leader and one follower will remain connected at all times. One way to achieve this, regardless of the positions of the agents, is to chose $f(\delta)$ to be a function that is bounded above by $N \Delta$. For instance one could chose $f(\delta)=\frac{2 N \Delta}{\pi} \arctan (\delta)$.

Theorem 3: Let the closed loop dynamics of the system be given by (5) and (6). Assume that the communication graph $\mathcal{G}(t)$ is initially complete and that both (7) and (8) hold on $C o(\mathcal{G}(0) \cup d)$, where $d$ represents the coordinates of the goal. Then, $\mathcal{G}(t)$ remains complete for all $t \geq 0$.

\section{THE INCOMPLETE GRAPH CASE}

In this section, a special case of incomplete graphs is analyzed. It is still assumed that both the subset of leaders and the subset of followers initially make up complete graphs, but it is no longer assumed that all followers are connected to all leaders. Let $\mathcal{N}_{i}^{f}=\mathcal{N}_{i} \cap \mathcal{N}^{f}$ and $\mathcal{N}_{i}^{l}=\mathcal{N}_{i} \cap \mathcal{N}^{l}$ be the subsets of agent $i$ 's neighbors that belong to the group of followers and the group of leaders respectively, $\left|\mathcal{N}_{i}^{f}\right|=N_{f i}$, $\left|\mathcal{N}_{i}^{l}\right|=N_{l i}$. Thus, for each $i \in \mathcal{N}^{f}$ in the incomplete graph $\mathcal{N}_{i}^{f}=\mathcal{N}^{f}$ while $\mathcal{N}_{i}^{l} \subseteq \mathcal{N}^{l}$. For each $j \in \mathcal{N}^{l}$ we instead have $\mathcal{N}_{j}^{l}=\mathcal{N}^{l}, \mathcal{N}_{j}^{f} \subseteq \mathcal{N}^{f}$. The dynamics of follower $i$ are:

$$
\dot{x}_{i}=-\sum_{k \in \mathcal{N}^{f}}\left(x_{i}-x_{k}\right)-\sum_{k \in \mathcal{N}_{i}^{l}}\left(x_{i}-x_{k}\right),
$$

while the dynamics of leader $j$ are given by

$$
\dot{x}_{j}=-\sum_{k \in \mathcal{N}^{l}}\left(x_{j}-x_{k}\right)-\sum_{k \in \mathcal{N}_{j}^{f}}\left(x_{j}-x_{k}\right)+F\left(x_{j}, d\right) .
$$

We will begin the connectivity analysis by deriving sufficient conditions for the two initially complete subgraphs to remain complete. Then the links connecting the subgraphs are analyzed and a lower bound is determined for the number of leader-follower links needed to guarantee that a given link between a leader and a follower is kept intact.

follower-follower connections: Considering the connection between two followers $i, j \in \mathcal{N}^{f}$. Both followers have links to all other follower agents and possibly, but not necessarily, links to some or all of the leader agents. The sets $\mathcal{N}_{i}^{l}$ and $\mathcal{N}_{i}^{l}$ may be disjunct or overlapping. Define $\mathcal{N}_{c}^{l}=\mathcal{N}_{i}^{l} \cap \mathcal{N}_{j}^{l} \subseteq \mathcal{N}^{l}$ to be the set of leaders that followers $i$ and $j$ have in common, $\left|\mathcal{N}_{c}^{l}\right|=N_{l c}$. Note that if $k \in \mathcal{N}_{i}^{l}$ 
then $\left|x_{i}-x_{k}\right| \leq \Delta$. For the two followers we get

$$
\begin{aligned}
\frac{d \delta_{i j}^{2}}{d t}= & 2\left(x_{i}-x_{j}\right)^{T}\left[-N_{f}\left(x_{i}-x_{j}\right)-N_{l c}\left(x_{i}-x_{j}\right)\right. \\
& \left.-\sum_{k \in \mathcal{N}_{i}^{l} \backslash \mathcal{N}_{c}^{l}}\left(x_{i}-x_{k}\right)+\sum_{k \in \mathcal{N}_{j}^{l} \backslash \mathcal{N}_{c}^{l}}\left(x_{j}-x_{k}\right)\right] \\
= & -2\left(N_{f}+N_{l c}\right) \delta_{i j}^{2} \\
& +2\left(x_{i}-x_{j}\right)^{T}\left[\sum_{k \in \mathcal{N}_{j}^{l} \backslash \mathcal{N}_{c}^{l}}\left(x_{j}-x_{k}\right)\right. \\
& \left.-\sum_{k \in \mathcal{N}_{i}^{l} \backslash \mathcal{N}_{c}^{l}}\left(x_{i}-x_{k}\right)\right] \\
\leq \quad & 2\left(N_{f}+N_{l c}\right) \delta_{i j}^{2} \\
& +2\left(N_{l i}+N_{l j}-2 N_{l c}\right) \Delta \delta_{i j} .
\end{aligned}
$$

Note that $\left(N_{l i}+N_{l j}-2 N_{l c}\right) \geq 0$. Requiring $\frac{d \delta_{i j}^{2}}{d t} \leq 0$ when $\delta_{i j}=\Delta$ leads to the constraint $N_{l i}+N_{l j} \leq N_{f}+3 N_{l c}$, which is satisfied for all follower-follower connections, regardless of the topology, for every graph that has

$$
N_{f} \geq N_{l} \text {. }
$$

leader-leader connections: Consider now the connection between two arbitrary leaders $i, j \in \mathcal{N}^{l}$. Following the notation in the follower-follower case, we let $\mathcal{N}_{c}^{f}=\mathcal{N}_{i}^{f} \cap$ $\mathcal{N}_{j}^{f} \subseteq \mathcal{N}^{f}$ be the set of followers that $i$ and $j$ have in common, $\left|\mathcal{N}_{c}^{f}\right|=N_{f c}$. We get

$$
\begin{aligned}
\frac{d \delta_{i j}^{2}}{d t} \leq & -2\left(N_{l}+N_{f c}\right) \delta_{i j}^{2}+2\left(N_{f i}+N_{f j}-2 N_{f c}\right) \Delta \delta_{i j} \\
& +2\left(x_{i}-x_{j}\right)^{T}\left[F\left(x_{i}, d\right)-F\left(x_{j}, d\right)\right] .
\end{aligned}
$$

We shall investigate the case $\delta_{i j}=\Delta$ closer. Define $\alpha$ to be the angle between $j$ and $d$, as seen by $i$. Then, for an arbitrary choice of $x_{i}$ we have $x_{j}=x_{i}+\Delta \mathbf{e}$, where $\mathbf{e}=\frac{x_{j}-x_{i}}{\Delta}$. The dependence between $\delta_{i}$ and $\delta_{j}$ follows from the law of cosines, such that for a given $\alpha$ we get $\delta_{j}=$ $\sqrt{\Delta^{2}+\delta_{i}^{2}-2 \Delta \delta_{i} \cos \alpha}$. Without loss of generality we can assume that $\delta_{i} \geq \delta_{j}$, and that consequently $-\frac{\pi}{2}<\alpha<\frac{\pi}{2}$ and $\cos \alpha \geq 0$. For the moment we also assume that $\delta_{j}>0$.

With $\delta_{i j}=\Delta$, (12) becomes

$$
\begin{aligned}
\frac{d \delta_{i j}^{2}}{d t} \leq & 2\left(N_{f i}+N_{f j}-3 N_{f c}-N_{l}\right) \Delta^{2} \\
& +2\left(x_{i}-x_{j}\right)^{T}\left[F\left(x_{i}, d\right)-F\left(x_{j}, d\right)\right] . \\
\leq & 2\left(N_{f}-N_{l}\right) \Delta^{2} \\
& +\underbrace{2\left(x_{i}-x_{j}\right)^{T}\left[F\left(x_{i}, d\right)-F\left(x_{j}, d\right)\right]}_{\gamma} .
\end{aligned}
$$

and with $x_{j}=x_{i}+\Delta \mathbf{e}$, the term $\gamma$ becomes

$$
\begin{aligned}
\gamma & =-2 \Delta e^{T}\left[\left(\frac{f\left(\delta_{i}\right)}{\delta_{i}}-\frac{f\left(\delta_{j}\right)}{\delta_{j}}\right)\left(d-x_{i}\right)+\frac{f\left(\delta_{j}\right)}{\delta_{j}} \Delta e\right] \\
& =-2 \Delta \underbrace{\left(\frac{f\left(\delta_{i}\right)}{\delta_{i}}-\frac{f\left(\delta_{j}\right)}{\delta_{j}}\right)}_{(*)} \delta_{i} \cos \alpha-2 \frac{f\left(\delta_{j}\right)}{\delta_{j}} \Delta^{2} .
\end{aligned}
$$

Requiring $\frac{d \delta_{i j}^{2}}{d t} \leq 0$ leads to

$$
\underbrace{\left(\frac{f\left(\delta_{i}\right)}{\delta_{i}}-\frac{f\left(\delta_{j}\right)}{\delta_{j}}\right)}_{(*)} \delta_{i} \cos \alpha+\frac{f\left(\delta_{j}\right)}{\delta_{j}} \Delta \geq\left(N_{f}-N_{l}\right) \Delta .
$$

From condition (11) it follows that the right hand side of the equation is positive. Also, it is known that $f(\delta) \geq 0$. Thus, the only term in the above expression that may be negative is $(*)$. The sign of $(*)$ depends on the characteristics of $f(\delta)$ and may be constant or time varying. Due to page limitations we shall confine the analysis in this paper to the case $(*) \geq 0$.

Recall that, by assumption, $\delta_{i} \geq \delta_{j}$. Then $(*) \geq 0$ means that $f(\delta)$ must be a convex function, $f^{\prime}(\delta) \geq 0$. Under those conditions it is sufficient to require

$$
\frac{f\left(\delta_{j}\right)}{\delta_{j}} \geq N_{f}-N_{l}
$$

where $N_{f}-N_{l} \geq 0$ according to (11). Since $\frac{f(\delta)}{\delta}$ is monotonically increasing (which follows from the fact that $f(\delta)$ is a positive convex function) we can use l'Hôpitals rule to obtain a lower bound on $\frac{f(\delta)}{\delta}, \inf _{\delta>0} \frac{f(\delta)}{\delta}=\lim _{\delta^{+} \rightarrow 0} \frac{f(\delta)}{\delta}=f^{\prime}(0)$. This means that (13) is satisfied if

$$
f^{\prime}(0) \geq N_{f}-N_{l}
$$

In the special case where we have $\delta_{j}=0$, the condition corresponding to (13) becomes $\frac{f(\Delta)}{\Delta} \geq N_{f}-N_{l}$. This is obviously satisfied for all convex goal-attraction functions that satisfy (13).

Remark 1: By definition, linear functions are a special case of convex functions. For a linear function $f(\delta)=\beta \delta$, $\beta>0$, Eq. (13) can be simplified as $\beta \geq N_{f}-N_{l} \geq 0$.

leader-follower connections: Finally we derive what it takes to keep the leader and the follower subgraphs connected. Consider follower $i \in \mathcal{N}^{f}$ and leader $j \in \mathcal{N}^{l}$. Let $f_{\max }$ as before be defined as the largest value the goal attraction function $f\left(\delta_{j}\right)$ can assume within the convex hull of the initial positions of all agents in the network and the goal position $d$. While $x_{j} \neq d$ we have $\frac{d \delta_{i j}^{2}}{d t}=$ $2\left(x_{i}-x_{j}\right)^{T}\left[-\sum_{k \in \mathcal{N}^{f}}\left(x_{i}-x_{k}\right)-\sum_{k \in \mathcal{N}_{i}^{l}}\left(x_{i}-x_{k}\right)+\right.$ $\left.\sum_{k \in \mathcal{N}^{l}}\left(x_{j}-x_{k}\right)+\sum_{k \in \mathcal{N}_{j}^{f}}\left(x_{j}-x_{k}\right)-F\left(x_{j}, d\right)\right]$, which after some calculations yields

$$
\begin{aligned}
\frac{d \delta_{i j}^{2}}{d t}= & -2 N_{f j} \delta_{i j}^{2}-2 N_{l i} \delta_{i j}^{2}+2\left(x_{i}-x_{j}\right)^{T}\left[-F\left(x_{j}, d\right)\right. \\
& \left.-\sum_{k \in \mathcal{N}^{f} \backslash \mathcal{N}_{j}^{f}}\left(x_{i}-x_{k}\right)+\sum_{k \in \mathcal{N}^{l} \backslash \mathcal{N}_{i}^{l}}\left(x_{j}-x_{k}\right)\right] \\
\leq & -2\left(N_{f j}+N_{l i}\right) \delta_{i j}^{2}+2 f_{\max } \delta_{i j} \\
& +2\left(N_{f}-N_{f j}\right) \Delta \delta_{i j}+2\left(N_{l}-N_{l i}\right) \Delta \delta_{i j} .
\end{aligned}
$$

Setting $\frac{d \delta_{i j}^{2}}{d t} \leq 0$ when $\delta_{i j}=\Delta$ leads to

$$
\frac{N}{2}+\frac{f_{\max }}{2 \Delta} \leq N_{f j}+N_{l i}
$$

It is easy to see that if $x_{j}=d$, (15) is still a sufficient condition for $i, j$ to remain connected since the effect of 
setting $F\left(x_{j}, d\right)=0$ will be a relaxation of the inequality. Thus, if the leader and follower subgraphs remain complete and if (15) holds for all initial links between leaders and followers, all connections in the graph will be maintained.

The previous derivations are summarized in the following:

Theorem 4: Assume that the communication graph $\mathcal{G}(t)$ is initially connected and constituted of two complete subgraphs, the subgraph of leaders and the subgraph of followers. The dynamics of the follower and leader agents are given by (9) and (10) and the magnitude of the maximum goal attraction force that can be experienced by the leader agents is $f_{\max }=\max _{x \in C o(\mathcal{G}(0) \cup d)} f(|d-x|)$. Assume also that

(A1) $\mathcal{G}(t)$ satisfies condition (11),

(A2) $f(\delta)$ is a convex function on $C o(\mathcal{G}(0) \cup d)$ such that (14) is true,

(A3) (15) holds for all initial links $(i, j)$ such that $i \in$ $\mathcal{N}^{l}, j \in \mathcal{N}^{f}$.

Then all links in $\mathcal{G}(t)$ will be maintained for $t \geq 0$.

In some cases the following is also useful:

Lemma 5: Let $\mathcal{G}(t)$ be the communication graph in Theorem 4 and let $E^{*}$ be a subset of the initial links between the group of leaders and the group of followers. Re-define the neighbor sets of the graph such that $i \in \mathcal{N}^{f}$ is considered a neighbor of $j \in \mathcal{N}^{l}$, and vice versa, if and only if they are initially connected and the link $(i, j) \in E^{*}$. Then, if $(A 1)$ and $(A 2)$ are satisfied and it is possible to find a subset $E^{*}$, $\left|E^{*}\right| \geq 1$, such that condition (15) is satisfied for all links in $E^{*}$, then all links in $E^{*}$ will remain intact and the groups of leaders and followers will remain connected with each other.

Proof: See [2]. $\diamond$

\section{Simulations}

The results of Section IV are illustrated in a series of simulations. We consider a scenario where the subgraph of leaders and the subgraph of followers are complete but where the full graph is not. A linear attraction $f(\delta)=\beta \delta$ is used. Moreover, $\Delta=10$ and $d=\left(\begin{array}{ll}0 & 0\end{array}\right)$ in the examples.

1) Example 1: Stable graph: Consider first nine agents, four leaders and five followers. Let $\mathcal{G}(t)$ denote the timedependent communication graph, so that $\mathcal{G}(0)$ is decided by the initial configuration of the agents, shown in Fig. 1. Apparently $\mathcal{G}(0)$ is not complete. As seen in the figure, the rightmost of the followers is outside the sensing range of all the leaders while the reverse is true for the leftmost of the leaders. The remaining seven agents form a complete subgraph. Using $\beta=1$, both (11) and (14) are satisfied and condition (15) is true for all existing links between the leader and the follower group. According to Theorem 4, this guarantees that all links in the initial graph are maintained, a result that is confirmed by the simulation. The links are all kept intact as the robots converge to the goal position and as the agents move closer to each other new links are formed, one by one, until the graph is complete. The trajectories of the converging robots are shown in Fig. 2.

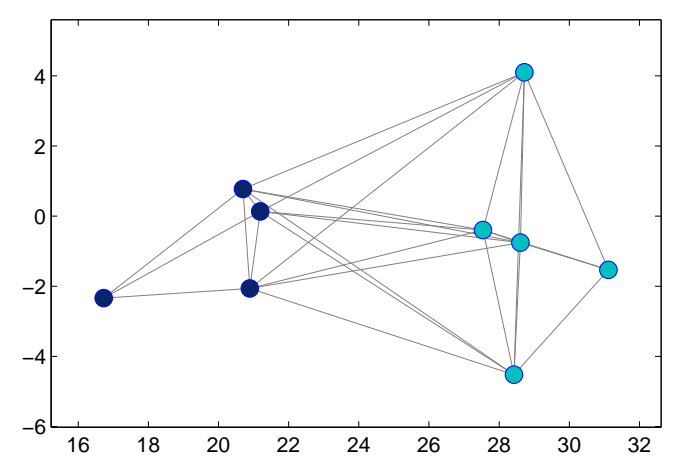

Fig. 1. Initial configuration of the agents in Example 1 and 2. Leaders and followers are represented by darker and lighter dots.

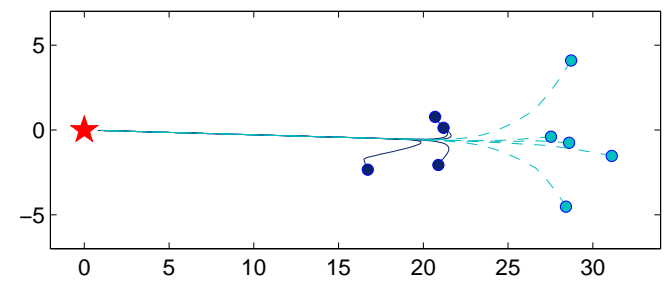

Fig. 2. Example 1: Trajectories of robots converging to the goal.

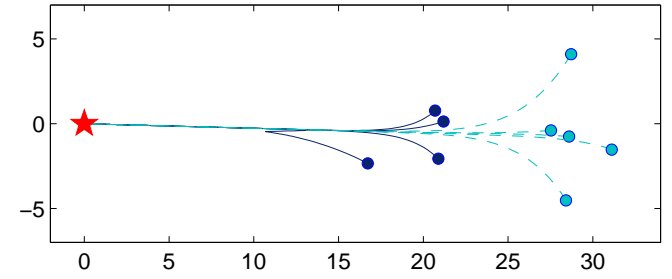

Fig. 3. Example 2: The initial positions of the robots are the same as in Example 1 but the attraction to goal is stronger and condition (15) that guarantees preservation of leader-follower links is violated.

2) Example 2: Leader-to-follower stability: If the same simulation is run with a larger value on $\beta(\beta=2)$, the influence of $f(\delta)$ increases. Conditions (11) and (14) are still satisfied but the inequality in (15) is no longer true for any of the existing leader-follower links and it is not guaranteed that the connection between the leader and the follower subgroup is kept. The simulation shows that all agents still converge towards the goal (see Fig. 3), but because of the stronger attractive force, the leaders move faster towards $d$ and as a result, one of the leader-follower links is temporarily broken during the first part of the simulation. In this example, only one link was broken and it was later re-formed, but in order to predict this fortunate outcome of the simulation a thorough analysis of the system would have been needed. Making sure that condition (15) is satisfied presents an easy way of avoiding the undesirable situation where the leader and the follower agents lose contact with each other.

3) Example 3: Leader-to-leader stability: Let us consider Fig. 4. Except for the single leader to the left, all agents are within sensor range of each other. With this setup we can 


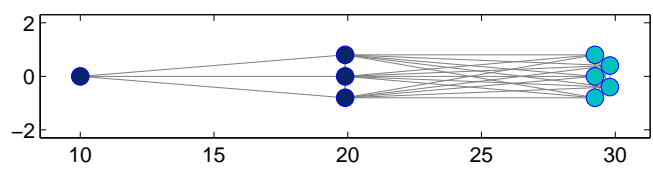

Fig. 4. Initial configuration of the agents in Example 3.

choose $\beta$ such that (11) and (15) are true while (14) is violated, and such that the leftmost of the leaders loses contact with the rest of the agents. Fig. 5 shows the trajectories of the agents in a simulation where $\beta=0.02$ while Fig. 6 shows the separation between the disconnected leader and the rest of the group as a function of time. Since all leaders know the coordinates of the goal, all agents eventually re-connect as they are approaching $d$. The follower agents on the other hand are depending on the leaders for navigation, so it is more serious if one of the followers loses contact with the group. This scenario is treated in the final example.

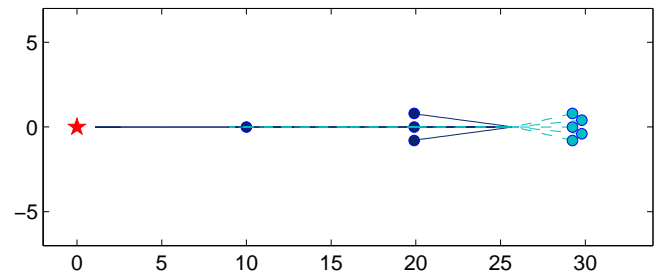

Fig. 5. Example 3: Due to the weakness of the goal attraction force, condition (14) is violated and the single leader closest to the goal temporarily loses contact with the group.

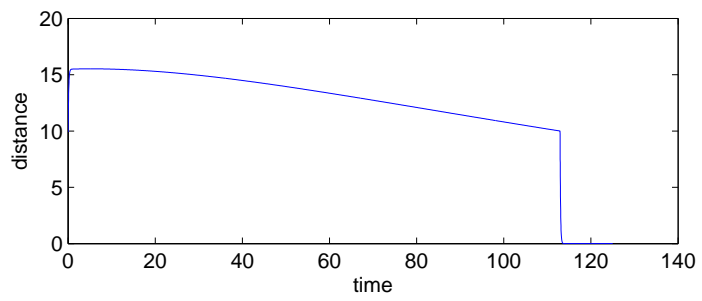

Fig. 6. Distance separating the disconnected leader in Example 3.

4) Example 4: Follower-to-follower stability: To illustrate the importance of maintaining the links within the follower subgroup we study a graph consisting of four followers and five leaders. The initial positions are shown in Fig. 7. Clearly, the ratio of leaders-to-followers in the graph violates (11), but with a proper choice of $\beta$ it is possible to satisfy both (14) and (15) for all leader-follower links.

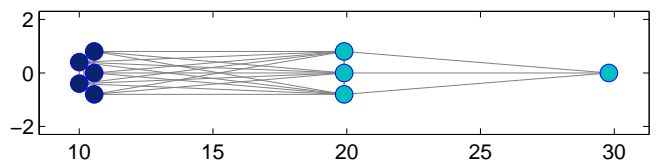

Fig. 7. Initial configuration of the agents in Example 4.

The simulation was run with $\beta=1$ and the result is shown in Fig. 8. In the initial setup, the rightmost of the followers is connected to the rest of the group only through links to the other followers. These links are broken short after the simulation is started and once the contact to the other agents is lost, the disconnected follower has no way of localizing neither the other agents nor the goal. As a result, the lost follower is left behind and it never reaches the goal.

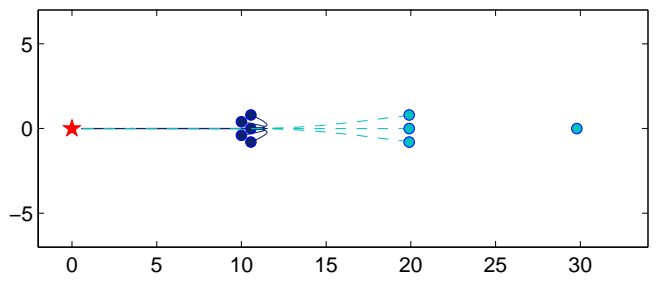

Fig. 8. Example 4: Condition (11), that guarantees the completeness of the follower subgroup, is violated. The single follower to the right loses contact with the group permanently short after the start of the simulation.

\section{CONCLUSIONS}

We examined how the network structure affects connectivity and convergence to a common goal for a group of agents in a leader-follower multi-agent network with proximity based communication topology. The geometric approach is demonstrated on two networks with special structure on the initial topology. In the first example, we studied a network where the communication graph was initially complete. In the second example we considered an incomplete network consisting of two complete subgraphs that were linked together. Further research includes an investigation on the types of information structures on which this approach is useful.

\section{REFERENCES}

[1] M. Arcak. Passivity as a design tool for group coordination. IEEE Transactions on Automatic Control, 52(8):1380-1390, 2007.

[2] D.V. Dimarogonas, T. Gustavi, M. Egerstedt, and X. Hu. On the number of leaders needed to ensure network connectivity. 47th IEEE Conf. Decision and Control, pages 1797-1802, 2008.

[3] C. Godsil and G. Royle. Algebraic Graph Theory. Springer Graduate Texts in Mathematics \# 207, 2001.

[4] M. Ji and M. Egerstedt. Distributed coordination control of multiagent systems while preserving connectedness. IEEE Transactions on Robotics, 23(4):693-703, 2007.

[5] S.G. Loizou and K.J Kyriakopoulos. Navigation of multiple kinematically constrained robots. IEEE Transactions on Robotics, 24(1):221231, 2008.

[6] S. Martinez, F. Bullo, J. Cortes, and E. Frazzoli. On synchronous robotic networks - Part I: Models, tasks and complexity. IEEE Transactions on Automatic Control, 52(12):2199-2213, 2007.

[7] R. Olfati-Saber and R.M. Murray. Consensus problems in networks of agents with switching topology and time-delays. IEEE Transactions on Automatic Control, 49(9):1520-1533, 2004.

[8] A. Rahmani, M. Ji, M. Mesbahi, and M. Egerstedt. Controllability of multi-agent systems from a graph-theoretic perspective. SIAM Journal on Control and Optimization, 48(1):162-186, 2009.

[9] B. Smith, J. Wang, M. Egerstedt, and A. Howard. Automatic formation deployment of decentralized heterogeneous multiple-robot networks with limited sensing capabilities. IEEE International Conference on Robotics and Automation, 2009. to appear.

[10] H.G. Tanner. On the controllability of nearest neighbor interconnections. 43rd IEEE Conference on Decision and Control, pages 24672472, 2004. 